\title{
NORMAS OBTIDAS NA INSTALAÇÃO DO CENTRO DE EDUCAÇÃO E ALIMENTAÇÃO DO PRÉ-ESCOLAR (CEAPE) EM GRUPO ESCOLAR DO MUNICf́PIO DE SÃO PAULO, BRASIL *
}

\author{
Maria Lúcia Ferrari CAVALCANTI ** \\ Avany Maria Xavier BON ** \\ Maryland MIGUEL ** \\ Yaro Ribeiro GANDRA **
}

RSPU-B/208

Cavalcanti, M. L. F. et al. - Normas obtidas na instalação do Centro de Educação e Alimentação do Pré-Escolar (CEAPE) em grupo escolar do municipio de São Paulo, Brasil. Rev. Saúde públ., S. Paulo, 8:93-103, 1974.

RESUMo: Para extrair normas que possam servir de guia para a instalação de Centros de Educação e Alimentação do Pré-Escolar (CEAPE) em escolas da Capital, foi instalado o CEAPE em Grupo Escolar da Prefeitura do Municipio de $\$ \tilde{a} o$ Paulo, Brasil. As normas obtidas referem-se: a) ao treinamento em Nutriçấo de professores do Grupo Escolar, responsáveis pelo programa; b) ao Curso de Nutrição ministrado às mães que inscreverem seus filhos pré-escolares no $C E A P E, e$ c) à instalação propriamente dita do programa, referente à freqüência dos pré-escolares e à educação nutricional das mães, através de sua participação nas atividades do CEAPE. Recomendase a motivação preliminar do Diretor e dos professores do Grupo Escolar para garantir a continuidade do programa; o conhecimento prévio das características da comunidade e da escola para adaptação do CEAPE às condiçōes locais. Foi preconizada a extensāo dos Centros de Educação e Alimentacẫo do PréEscolar através das redes de escolas primárias, como medida eficiente e econômica para melhorar as condições de saúde do pré-escolar.

*

Unitermos: Alimentação*; Pré-escolares, São Paulo (Brasil) *; Educação nutricional*; Saúde escolar.

O Centro de Educação e Alimentação do Pré-Escolar (CEAPE) ** tem como objetivo a extensão da educação e assistência alimentar ao pré-escolar, de preferência irmão do escolar matriculado, e inclui como característica, a participação ativa e

* Trabalho apresentado no $6 .^{\circ}$ Congresso Brasileiro de Nutricionistas, $3 .^{\circ}$ Congresso Brasileíro de Nutrição e 1.a Reuniāo Brasileira sobre a Formação de Nutricionistas, São Paulo, 1972.

** Do Departamento de Nutrição da Faculdade de Saúde Pública da USP - Av. Dr. Arnaldo, 715 - Sảo Paulo, SP - Brasil.

** Plano para Assisténcia Alimentar do Pré-escolar, apresentado pelo Prof. Yaro Ribeiro Gandra, no $X$ Congresso Nacional de Estudos da CNAE, realizado em São Paulo, 1972. 
CAVALCANTI, M. L. F. et al. - Normas obtidas na instalação do Centro de Educação e Alimentação do Pré-Escolar (CEAPE) em grupo escolar do municipio de Săo Paulo, Brasil. Rev. Saúde puibl., S. Paulo, 8: 93-103, 1974.

a educação da mãe da criança assistida. E um programa de governo que utiliza recursos humanos e materiais pré-existentes, a ser desenvolvido nas redes de escolas primárias, aproveitando suas instalações, espaços ociosos e pessoal. Ainda mais, aproveita todo sistema administra tivo que se incumbe de prover a merenda escolar de gêneros alimentícios. A participação obrigatória das mães no CEAPE é uma característica fundamental do programa.

Dentro da nossa conjuntura econômico-social, a criação de Centros de Educação e Alimentação do Pré-Escolar constitui medida eficiente e econômica que se destina a atender uma população que, indiscutivelmente reclama por prioridade em programas de Nutrição: o Pré-Escolar.

\section{Objetivo}

Com a finalidade de extrair normas que pudessem servir de guia para a implantação de Centros de Educação e Ássistência Alimentar do Pré-Escolar em escolas da Capital, instalamos o CEAPE em Grupo Escolar da Prefeitura do município de São Paulo, contando com a colaboração do Departamento de Assistência Escolar daquela Prefeitura.

0 objetivo do presente trabalho é, pois, apresentar as primeiras normas obtidas na instalação desse Centro.

\section{MATERIAI E METODOS}

Foi escolhido para instalação do CEAPE um Grupo Escolar de bairro da periferia do município de São Paulo, com 1.039 alunos, e o cadastramento parcial dos préescolares, feito preliminarmente, revelou a existência de 371 crianças na faixa de 2 a 6 anos.

A metodologia utilizada na implantação do programa incluiu: a) treinamento em Nutrição de professores do Grupo Escolar que participassem das atividades do CEAPE;

b) curso de Nutrição para as mães que inscrevessem seus filhos no programa;

c) instalação propriamente dita do CEAPE, com a análise da freqüência das crianças e da participação ativa das mães.

RESULTA DOS

No que concerne à primeira fase da instalação do Centro de Educação e Assistência Alimentar do Pré-Escolar, a direção do estabelecimento recebeu circular com informações sobre o CEAPE, onde também se fixavam os compromissos assumidos pela escola (Anexo 1), conseguindo-se assim a motivação desejada. Foi então ministrado aos professores um curso teóricoprático que totalizou 20 horas, no qual foram abordados temas básicos como a alimentação e saúde, nutrientes e alimen tos, dieta adequada e conseqüências de uma alimentação defeituosa. Deu-se enfoque especial ao pré-escolar, examinando-se suas exigências nutritivas e fatores que concorrem para as deficiências alimentares desse grupo vulnerável. As responsabilidades do pessoal do Grupo Escolar participante do CEAPE, o trabalho do professor no treinamento em serviço da mãe, e os métodos de avaliação do programa também constituíram objeto de estudo. 0 programa desenvolvido apresentamos no Anexo II. Ao término do curso procedeu-se à avaliação escrita, sob forma de testes.

Em prosseguimento ao trabalho, foram convocadas para uma palestra as mães que desejassem inscrever seus pré-escolares no programa. 0 objetivo dessa palestra foi dar esclarecimentos sobre o CEAPE e, ao 


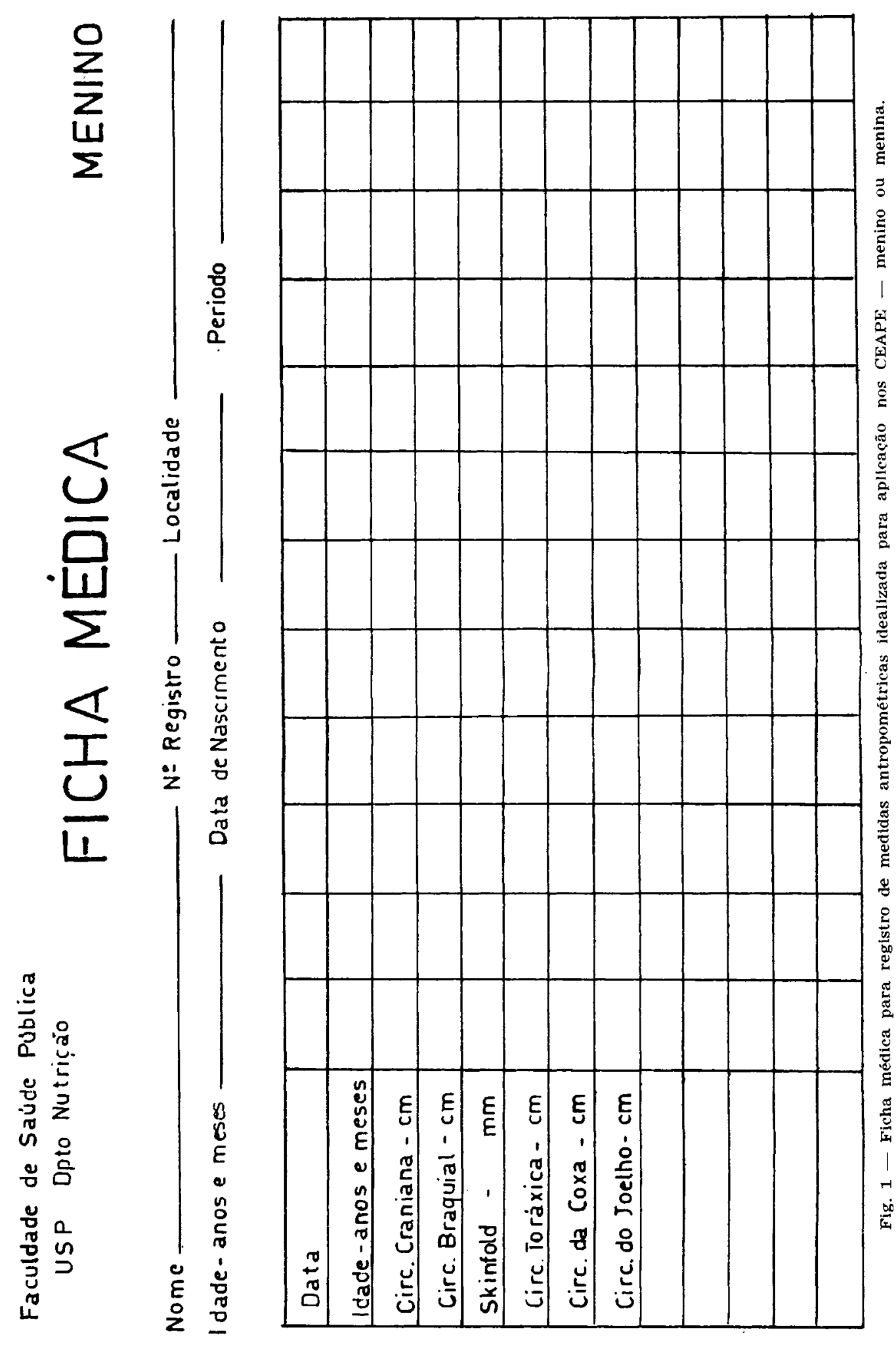




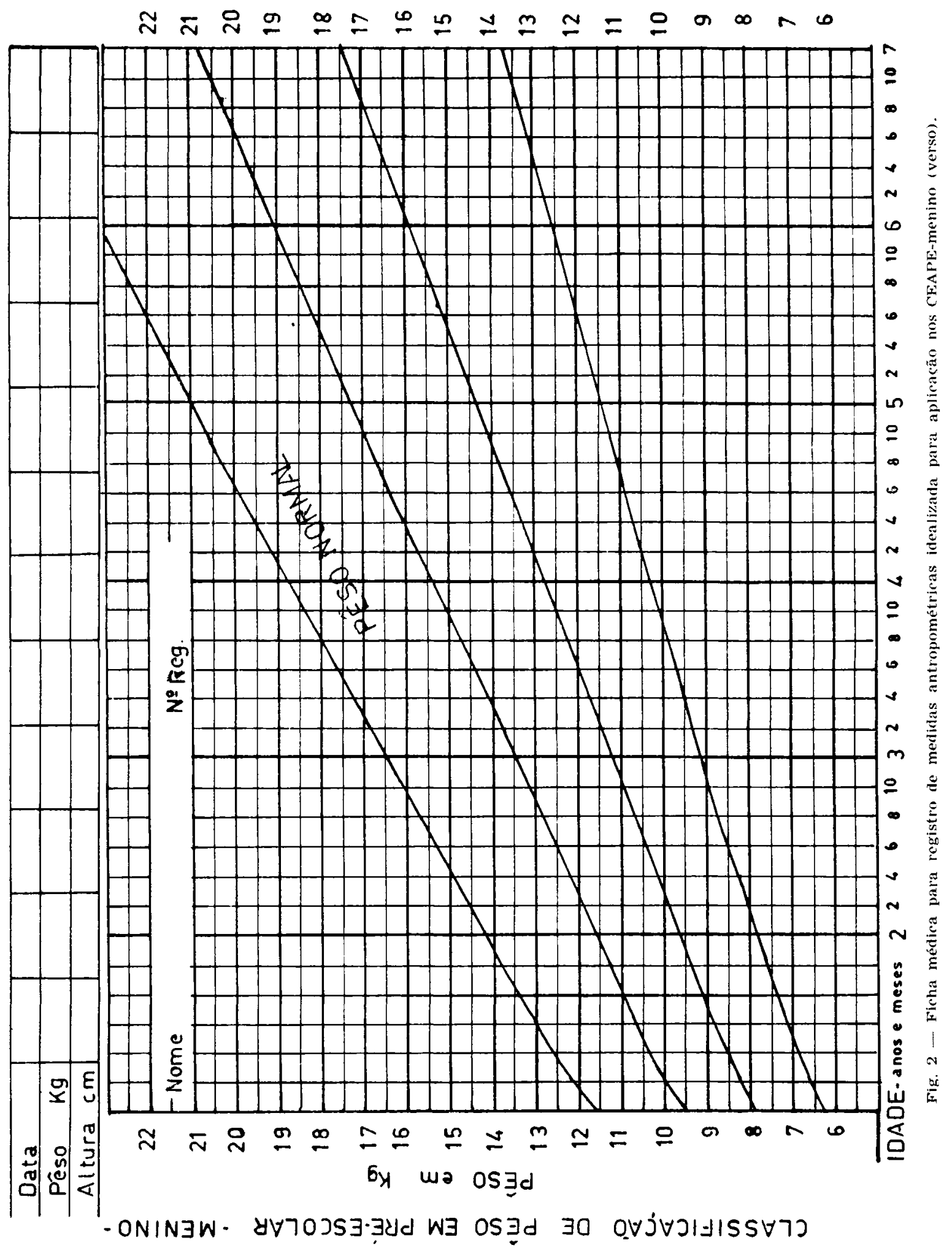




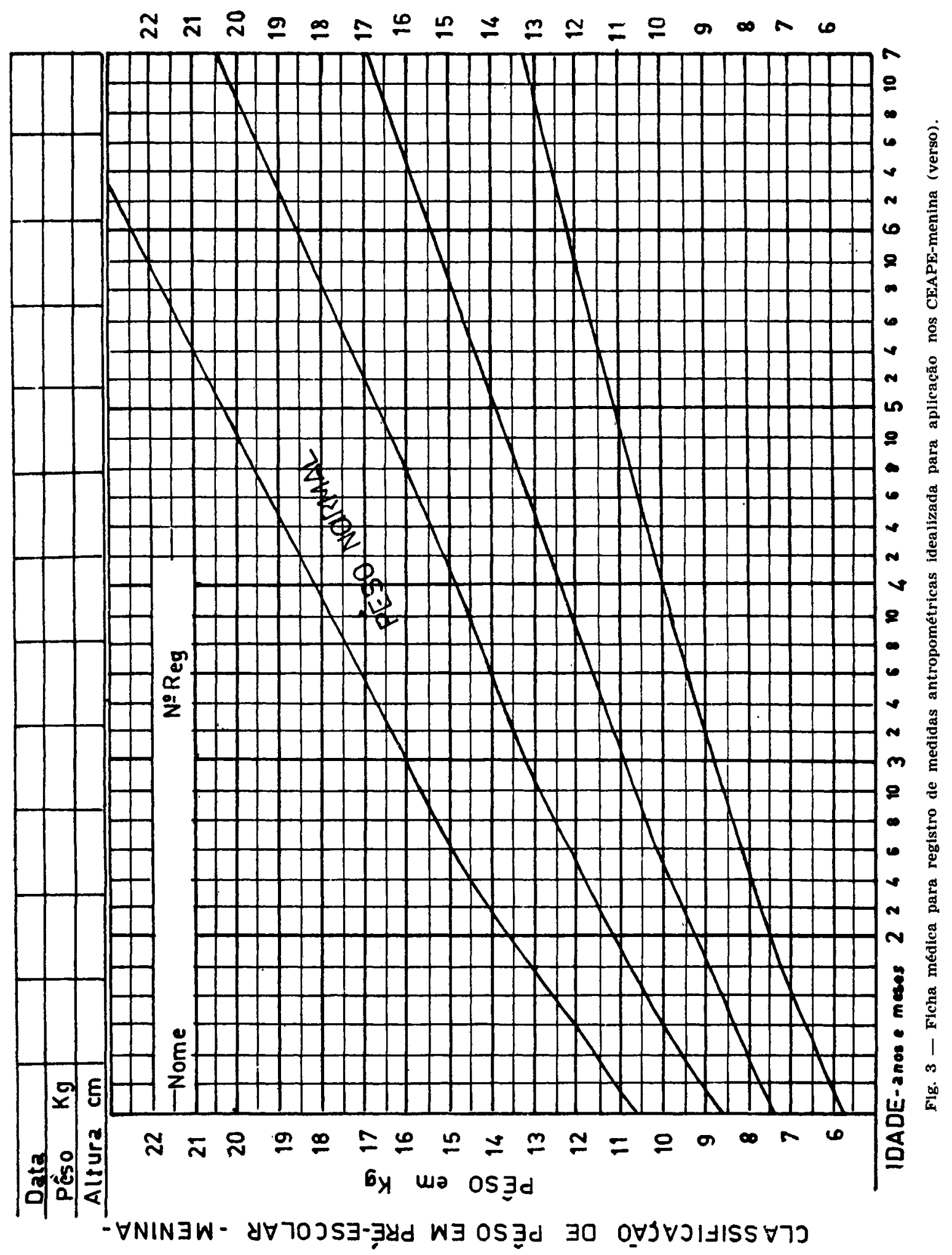


CAVALCANTI, M. L. F. et al. - Normas obtidas na instalação do Centro de Educação e Alimentaçáo do Pré-Escolar (CEAPE) em grupo escolar do municipio de Sáo Paulo, Brasil. Rev. Saúde públ., S. Paulo, 8: 93-103, 1974.

mesmo tempo, motivar as mães para contar com sua participação.

As mães que se inscreveram $(40)$ assinaram um termo de compromisso com o programa (Anexo III), e opinaram sobre o melhor período para a realização do curso a elas destinado.

O curso de Nutrição para as mães totalizou 10 horas de aulas teórico-práticas. A parte teórica incluiu noções básicas de Nutrição, o papel da mãe na formação de hábitos higiênicos e alimentares adequados para a promoção da saúde, características do pré-escolar e suas necessidades nutritivas; organização e aspectos de higiene no trabalho da mãe merendeira, suas responsabilidades e deveres. A parte prática constou de demonstrações do preparo de merendas na cozinha do Grupo Escolar, familiarizando as mães com as atividades que realizariam no CEAPE. $O$ anexo IV apresenta o programa desenvolvido.

Terminado o curso procedeu-se à determinação de peso e altura dos 60 pré-escolares inscritos, e foi distribuída a escala de comparecimento das mães ao Grupo Escolar. Foram também determinadas as seguintes medidas antropométricas dos préescolares: skinfold, circunferência braquial e circunferência craniana. As Figuras 1, 2, 3 (ficha médica e gráficos) constituem o Anexo V.

A freqüência das mães e das crianças ao CEAPE foi registrada diariamente pelos professores responsáveis. $\mathrm{Na}$ falta de crachás, os pré-escolares traziam presa à roupa identificação improvisada pelas mães.

A instalação propriamente dita do CEAPE efetivou-se de início em três períodos, participando 70 pré-escolares e 40 mães. Posteriormente, o programa passou a funcionar em dois periodos, atingindo 123 o número de crianças, com 68 mães inscritas.
As atividades das mães escaladas foram desenvolvidas, alternadamente, na cozinha (preparo de merenda e limpeza do material), no refeitório (distribuição de merenda) e junto às crianças, auxiliando em sua assistência e inclusive na recreação. As mães foram supervisionadas pelos professores treinados.

Por sugestão da equipe instaladora do CEAPE, foram aproveitados alunos dos 4. ${ }^{\circ}$ anos da escola que colaboraram na assistência aos pré-escolares.

\section{O M E N TA R O S}

$O$ treinamento em Nutrição dos professores que participarão do CEAPE é de fundamental importância, porquanto, a eles caberá a supervisão direta das mães durante sua atuação no programa. Por outro lado, serão os professores responsáveis por todas as atividades do CEAPE na escola, como por exemplo, a compra dos gêneros suplementares com recursos oferecidos pelos órgãos oficiais ou pela comunidade. E evidente que, com conhecimento em Nutrição, a seleção dos alimentos será mais adequada, como pudemos constatar no Grupo Escolar em que trabalhamos.

No que diz respeito ao Curso de Nutrição para as mães, o número de participantes e a frequiência das mesmas, foi excelente.

Para elaboração da escala de comparecimento e participação ativa das mães no CEAPE, foram consideradas as suas opções sobre os periodos de trabalho de sua maior conveniência. As mães que trabalhavam fora do lar propuseram-se a comparecer nos seus dias de folga.

A participação das mães no CEAPE foi efetiva e obedeceu à escala elaborada, evidenciando o interesse da comunidade pelo programa e sua aceitação. 
CAVAlCANTI, M. L. F. et al. - Normas obtidas na instalação do Centro de Educação e Alimentação do Pré-Escolar (CEAPE) em grupo escolar do município de São Paulo, Brasil. Rev. Saide pübl., S. Paulo, 8: 93-103, 1974

0 relacionamento escolares-pré-escolares não causou problemas. Devido a circunstâncias específicas do Grupo Escolar é que foi modificado o horário do CEAPE, que passou a funcionar em dois períodos. para evitar sobrecarga de trabalho no preparo e distribuição da merenda.

CONCLLSOES

Da instalação do CEAPE em Grupo Escolar da Prefeitura do Município de São Paulo, extraímos as seguintes normas que poderão servir para orientar a extensão futura do programa:

1 - é necessária a integração dos instaladores do programa com o serviço que promove a assistência alimentar na escola. e destes com a direção do estabelecimento;

2 - é eficiente a seqüência que experimentamos na instalação do CEA$\mathrm{PE}$ :

a) treinamento em Nutrição dos professores do Grupo Escolar. responsáveis pelo programa;

b) curso de Nutrição para as mães que inscreveram seus filhos no CEAPE;

c) instalação propriamente dita do programa, com assistência e educação alimentar das crianças, e educação das mães através de sua participação ativa:

3 - o treinamento em Nutrição dos professores é exigido porque a estes caberá a supervisão direta das mães durante sua atuação. Deverá ser feita imediata avaliação desse treinamento:

4 - a educação alimentar das mães far-se-á em processo contínuo;
5 - o treinamento em Nutrição. de preferência, deve ser feito por nutricionista. Entretanto, na impossibilidade de se contar com este técnico. sugerimos a preparação de um folheto que servirá de guia ao professor e à formação de agentes multiplicadores;

6 - a assinatura do termo de compromisso pelas mães é bastante útil, pois imprime maior valorização do programa por parte da comunidade, permite melhores condiçóes para planejamento do curso, facilitando também a elaboração da escala para comparecimento das mães ao CEAPE;

7 - a elaboração da escala de comparecimento das mães ao CEAPE deve levar em conta não só período de trabalho mais conveniente para a mãe como, também, o progressivo aumento do número de participantes do programa;

8 - são dados significativos para araliação do programa o registro de freqüência da mãe e do interesse por ela demonstrado durante sua participação. Esses dados serão registrados pelo professor responsável pelo CEAPE no Grupo Escolar;

9 - é indispensável um livro para registro da frequêencia do pré-escolar, bem como ficha individual para anotação periódica do peso. altura e demais medidas antropométricas das crianças. Caberá ao professor a obtenção e registro desses dados;

10 - é recomendável a motivação preliminar do diretor e dos professores da escola, para garantir a continuidade do programa. 0 
CAVAlCANTI, M. L. F. et al. - Normas obtidas na instalação do Centro de Educação e Allmentação do Pré-Escolar (CEAPE) em a. upo ej.olä do município de São Paulo, Brasil. Rev. Saúde pübl., S. Paulo, 8: 93-103, 1974.

CEAPE deverá ser apresentado com detalhes, e ficará estabelecido que o mesmo constitui programa da Escola e da comunidade;

11 - por ser o CEAPE um programa não rígido, que se adapta às características da escola e da comunidade, é recomendável um conhecimento prévio dessas condições para melhor ser estruturada sua instalação;

12 - é recomendável a extensão do
CEAPE, através das redes de escolas primárias, como medida eficiente e econômica para atingir a pré-escolar e melhorar as suas condições de saúde.

\section{A G R A D C I M E N T O}

Ao Doutor Cornélio Pedroso Rosenburg. Diretor do Departamento de Assistência Escolar da Prefeitura Municipal de São Paulo, pela colaboração e fornecimento de merendas.

Cavalcanti, M. L. F. et al. - [The norms obtained in the installation of Preschool Food Education Center (CEAPE) in a primary school of the city of S. Paulo, Brazil]. Rev. Saúde públ., S. Paulo, 8:93-103, 1974.

SUMMARY: To establish norms for the orientation and installation of Preschool Food Education Centers (CEAPE) in a school of the Capital, was the CEAPE in a primary municipal school of the city of São Paulo, Brasil. The norms obtained are refered to: a) Nutrition training for primary school teachers responsable for the program. b) Course in Nutrition lectured to mothers, that have enrolled their preschool children in the CEAPE. c) The effective installation of the program refered to preschool children frequency; and nutritional education of mothers, through their participation in the CEAPE activities. It was recommended the preliminary motivation of the director and teachers of the primary school to guarantee continuity of the program and also the previous knowledge of characteristics of the community and schools in order to adapt the CEAPE to local conditions. It was recommended that $C E A P E$ should be extended to primary school network as an efficient and economic measure to improve the preschool child's health conditions.

UNITERMS: Food*; Preschool children, S. Paulo (Brazil)*; Nutrition. educational*; School health.

\section{REFERENCIA BIBLIOGRAFICA}

1. GANDRA, Y. R. - Plano para a assistência alimentar do pré-escolar: Centro de Educaço e Alimentacio do Pré-escolar. Sāo Paulo, Faculdade de Saúde
Pública da USP. Departamento de Nutricão, 1972. [mimeografado].

Recebido para publicação em 18-12-1973. Aprovado para publicacaio em 21-1-197\%. 
CAVAlCaNTI, M. L. F. et al. - Normas obtidas na instalação do Centro de Educação e Alimentação do Pré-Escolar (CEAPE) em grupo escolar do municipio de são Paulo. Brasil. Rev. Saúde públ., S. Paulo, 8: 93-103, 1974.

\section{Axexo I}

Informargões sobre o CE.APE a Diretoria

1. O CEAPE é um programa gratuito. que visa orientar e esclarecer a mãe do pré-escolar sobre a importância da alimentação, através de participação ativa.

2. Exige a colaboração das mães em tudo que se relacione com "Merenda Escolar" fornecida ao pré-escolar.

3. A escola fornecerá merenda às crianças de 2 a 6 anos. irmãos dos escolares do Grupo, cujas mães se comprometem a colaborar com seu trabalho e a assistir às aulas sobre alimentação.

4. As mães que não comparecerem nos dias em que forem escaladas. terão seus filhos desligados do programa.

5. A diretora, a assistente pedagógica (AP), as auxiliares administrativas e as substitutas eventuais assistirão o curso sobre Nutrição. que lhes permitirá orientar as mães durante seu trabalho no Grupo.

6. Qualquer inscrição nova de mães ou exclusões do programa deverão ser comunicadas semanalmente à Faculdade de Saúde Pública.

7. A diretoria deverá fornecer aos organizadores do CEAPE. relação dos funcionários do Grupo responsáveis pela supervisāo do programa.

\section{Axexo II}

Programa Desenvolvido no Treinamento em Nutrição de Professores Primários

1. Alimentação e Saúde

2. Nutrientes e Alimentos
a) os nutrientes
b) funções
c) diferença entre nutrientes e ali- mentos

3. Grupos de Alimentos
a) construtores
b) reguladores
c) energéticos

4. Dieta adequada e consequências de uma alimentação defeituosa (carências !

5. O pré-escolar
a) características
b) necessidades nutritivas
c) fatores que concorrem para as deficiências alimentares do pré- escolar
d) o CEAPE como programa de assistência alimentar e educação do pré-escolar. e educação ali- mentar das mães

6. Merenda Escolar
a) objetivos
b) características de composição da merenda

7. Responsabilidades dos participantes do CEAPE
a) diretor
b) professores
c) merendeiras
d) mães 
CAVALCANTI, M. L. F. et al. - Normas obtidas na instalação do Centro de Educação e Alimentação do Pré-Escolar (CEAPE) em grupo escolar do munícípio de São Paulo, Brasil. Rev. Saúde públ., S. Paulo, 8: 93-103, 1974.

8. O trabalho da professora no treinamento em serviço da mãe
a) cuidados com os alimentos
b) higiene pessoal da mãe
c) higiene na distribuição
d) higiene do local de trabalho
e) relacionamento humano entre os participantes do CEAPE

9. Métodos de avaliação do programa

a) do pré-escolar:

peso e altura

freqüência

b) da mãe:

freqüência

interesse, dedicação e participação ativa

visita domiciliar

c) das professoras:

imediata através de testes

supervisão do treinamento em serviço das mães

\section{Avexo III}

Informações sobre o CEAPE e

Compromissos Assumidos pelas Mães

Inscritas no Programa

1. O pré-escolar constitui o grupo de crianças que mais necessita de assistência alimentar.

2. O CEAPE é um programa de orientação alimentar à mãe do pré-escolar e de assistência alimentar a essas crianças.

3. O Grupo Escolar realizará, pois, um programa com a finalidade de esclarecer e orientar as mães sobre a importância da alimentação do pré-escolar. 0 nome desse programa é CEAPE.
4. O CEAPE, contando com a colaboração das mães. fornecerá merenda às crianças de 2 a 6 anos de idade, irmãos dos escolares matriculados neste Grupo Escolar.

5. As mães, para se inscreverem, deverão se comprometer a prestar serviços relacionados com a merenda durante $\ldots$ dias por . ... e e assistir as aulas que serão dadas sobre alimentação.

6. Só receberão merenda, as crianças cujas mães cumprirem o compromisso assumido de frequiência ao Grupo Escolar nos dias escolhidos. O não comparecimento injustificado da mãe fará com que a criança perca o direito ao programa.

Estou de acordo em participar do programa $C E A P E$ nas condições acima.

Data :

Assinatura da mãe:

\section{Axexo IV}

Programa de Educação Alimentar para Mães

1. Alimentação e saúde

Papel da mãe na formação de hábitos higiênicos e alimentares, adequados para promoção de saúde. Nutrientes e Alimentos - Grupos de Alimentos.

2. Grupo de alimentos reguladores e energéticos.

- Quais são, valor nutritivo, quantidades recomendadas, falsas crenças e tabus 
CAVALCANTI, M. L. F. et al. - Normas obtidas na instalação do Centro de Educação e Alimentação do Pré-Escolar (CEAPE) em grupo escolar do município de são Paulo, Brasil. Rev. Saude pribl., S. Paulo, 8: 93-103, 1974.

- Reconhecimento do alimento fresco (compra)

- Higiene, conservação, armazenamento

- Preparo: técnica correta

- Horta doméstica

3. Grupo dos alimentos construtores

- Quais são. valor nutritivo. quantidades recomendadas, falsas crenças e tabus

- Reconhecimento do alimento fresco (compra)

- Higiene, conservação. armazenamento

- Preparo: técnica correta
- Criação de pequenos animais

- Dieta adequada

4. Alimentação do pré-escolar e merenda

- Necessidades nutritivas

- Quantidades recomendadas de alimentos

- Falsas crenças e tabus

- Hábitos de higinene pessoal e de higiene alimentar

5. Organização e aspectos de higiene no trabalho da mãe.

O papel da mãe no CEAPE, seus deveres e responsabilidades. 